aegypti eine höhere Wirksamkeit, als nach der Wirksamkeit der Komponenten zu erwarten wäre. Nach graphischer Ermittlung der $\mathrm{ED}_{50}$ auf Log-Probit-Papier sind Gemische von Bromophos und Methylparathion gegen Stegonomyia fasciata (= Aedes aegypti) doppelt (bei $70 \%$ Bromophos zu $30 \%$ Methylparathion sogar fast dreimal) so wirksam, als der additiven Wirksamkeit der Komponenten entsprechen würde. Mischungen mit einem überwiegendem Anteil an Methylparthion wirken besser wie Methylparathion allein.

E. Sch.

\section{BIOLOGISCHE BEKAMPFUNG}

Spradbery, J. P. (1970): Host finding by Rhyssa persuasoria L. an Ichneumonid parasite of Siricid Woodweeps. Animal Behaviour, 18, 1, S. 103-114.

Die vorliegende Arbeit beschreibt in ausführlicher Weise das Verhalten des Rhyssa persuasoria-Weibchens bei der Tätigkeit des Aufsuchens von befallenem Holz. Die Untersuchungen erfolgten an Pinus silvestris L., die Larven von Sirex noctilio L., S. juvencus L. oder $S$. cyaneus $F$. enthielten. Die Beobachtungen wurden bei $25^{\circ}$ Celsius und $70 \%$ relativer Luftfeuchtigkeit, unter Verwendung von fluoreszierendem Licht vorgenommen. Die Eiablage erfolgt 1 bis $3 \mathrm{~mm}$ tief in das Holz. Uber $5 \mathrm{~mm}$ tiefe Bohrungen werden durch Reize, die aus dem Holzinnern stammen, verursacht, wobei die Oberfläche Risse in Rinde und Holz aufweist, wodurch die Stelle des Einsetzens des Legebohrers beeinflußt wird. Die Eiablagelöcher der Siriciden sind für den Parasiten uninteressant und veranlassen ihn nicht zum Bohren. Es wird weiter festgestellt, daß die Weibchen von Rhyssa persuasoria imstande sind, tote Wirte in den Stämmen aufzuspüren und zu parasitieren, wodurch bewiesen wird, daß der Schall nicht unbedingt ein Stimulans zum Einbohren ist und für die Wirtsauffindung nicht unbedingt notwendig ist. Interessant ist ferner die Feststellung, da $B$ die Bohrspäne anlockende Wirkung besitzen, die jedoch mit zunehmender Entfernung vom Wirt abnimmt. Nasses Bohrmehl veranlaßt mehr Bohrungen als trockenes. Drei bis vier Monate alte, gezüchtete Pilzsymbionten sind am anziehendsten, sie entsprechen der Reifung der Wirtslarve im Holz unter ähnlichen Umständen. Es wird festgestellt, daß die Holzart nicht von Einfluß auf die Bohrtätigkeit ist. Vergleiche des Symbionten mit verschiedenen häufigen Waldpilzen erwiesen die spezifische Anziehungskraft des Symbionten. Vergleiche von bestimmten Stereum- und Amylosterum-Arten mit dem Symbionten zeigten eine deutliche Bevorzugung für den Sirex juvencus-Symbionten, der wahrscheinlich den Amylosterum-Arten sehr verwandt ist. Wasser- und Ethanolextrakte von Bohrmehl stimulierten die Bohrtätigkeit. Wenn Siricidenlarven auf Bohrmehl- oder Pilzmedien gehalten wurden oder wenn Siricidenlarvendarminhalt und Chitinase dem Medium beigegeben wurden, konnte eine größere Anziehungskraft des Substrates festgestellt werden.

A. Sinreich

Brian, M. V. (1969): Male production in the ant Myrmica rubra L. - Insectes Sociaux, Paris, XVI, 4, pp. $249-268$.

10 oder höchstens 40 Arbeiter genügen, um den Eierertrag einer Königin auf den Maximalgrad, pro Woche auf durchschnittlich 30 bei $20^{\circ} \mathrm{C}$ konstanter Temperatur, ansteigen zu lassen. Arbeiter legen 0,12 bis 0,40 Eier jede Woche ab. Die Kolonien steigern die Eierzahl im Verhältnis zu ihrer Größe, außer wenn ihre Dichte sehr hoch (über 10 Arbeiter pro ml) ist. Männchen-Larven erreichen nach dem Winter, wenn sie von königinnenlosen Arbeitern großgezogen werden, eine bedeutendere Größe und überleben besser als sonst. - Eine Verzögerung in der Vermehrung der Königinnen vergrößert den Prozentsatz der Männchen bei der Nachkommenschaft. Bei einer Verzögerung von 6 Wochen ist die Zahl der Männchen propor- tional der Arbeiterzahl in der Kolonie, doch die Zahl der Weibchen ist von allen Typen unabhängig. - Die Männchen-Produktion wird erörtert in Beziehung zur Koloniegröße und der Kolonie-Reife.

A. Herfs (Köln)

\section{Kleine Mitteilungen}

\section{PERSONALIEN}

Professor Dr. GüNTher Becker, Direktor der Bundesanstalt für Materialprüfung in Berlin-Dahlem, wurde zum Ehrenmitglied der Gütegemeinschaft für Holzschutz und Bautenschutz Berlin ernannt. Ferner erfolgte seine Wiederwahl zum Vorsitzenden der Arbeitsgruppe Holzschutz des Internationalen Verbandes Forstlicher Forschungsanstalten, zum Präsidenten der International Research Group of Wood Preservation und zum Präsidenten der Deutschen Gesellschaft für Holzforschung.

Professor Dr. J. Franz wurde zum Direktor des Instituts für Biologische Schädlingsbekämpfung der Biologischen Bundesanstalt für Land- und Forstwirtschaft in Darmstadt ernannt.

Herrn Landesforstmeister Professor emer. Dr. Fritz ScHWERDTFEGER, ehemaligem Leiter der Abt, B der Niedersächsischen Forstlichen Versuchsanstalt in Göttingen, wurde vom Herrn Bundespräsidenten das Große Verdienstkreuz des Verdienstordens der Bundesrepublik Deutschland verliehen. Die hohe Auszeichnung wurde ihm am 22.12.1970 in einer Feierstunde in seinem ehemaligen Dienstgebäude vom Niedersächsischen Minister für Ernährung, Landwirtschaft und Forsten, Herrn Bruns, überreicht.

Deutsches Weinbau-Jahrbuch 1971. Herausgeber: Prof. Dr. Bruno Götz und Prof. Dr. Waldemar Madel. Waldkircher Verlagsgesellschaft. 22 . Jahrgang. 272 Seiten und Kalendarium.

Das bekannte Jahrbuch enthält eine Fülle interessanter Beiträge und Fachartikel aus den verschiedensten Gebieten des Weinbaues. U, a. einen Artikel von M. HeRING über "Die heutige Lage der Bekämpfung des Gefurchten Dickmaulrüßlers in den Rebanlagen der Deutschen Weinbaugebiete". Weiters von SchruFr: „Die Bedeutung der Fungizidspritzung im Weinbau für den Rote-Spinne-Befall." A. DIETER berichtet "Uber die Anwendung selektiv wirkender Herbizide in Ertragslagen". Von allgemeinem Interesse ist der Beitrag von K. DECKER "Vom Ursprung unseres Weinbaues". Zahlreiche Tabellen, Hinweise auf Pflanzenschutzmittel und Pflanzenschutzgeräte sowie Anschriftenverzeichnisse machen das Jahrbuch zu einem praktischen und willkommenen Nachschlagebuch für alle Gebiete des Weinbaues.

E. Sch.

Jahresbericht 1969 der Hessischen Lehr- und Forschungsanstalt für Wein-, Obst- und Gartenbau, Ingenieurschule Geisenheim am Rhein. Herausgegeben von dem Direktor Dr. P. Clavs. 1970. 144 Seiten.

Der Jahresbericht unterrichtet über die Tätigkeit der Institute für Weinbau, Rebenzüchtung und Rebenveredelung, Weinchemie und Getränkeforschung, Mikrobiologie und Biochemie, Obstbau, Gemüsebau, Zierpflanzenbau, Gartenarchitektur und Landschaftspflege, Bodenkunde und Pflanzenernährung, Botanik, Pflanzenkrankheiten, Technik, Betriebswirtschaft und Marktforschung sowie über den staatlichen Reblausbekämpfungsdienst.

E. Sch.

Das 23, Internationale Symposium über Pflanzenschutz findet am 4. Mai 1971 in Gent statt. 